\title{
Motif-Based Protein Sequence Classification Using Neural Networks
}

\author{
KONSTANTINOS BLEKAS, DIMITRIOS I. FOTIADIS, and ARISTIDIS LIKAS
}

\begin{abstract}
We present a system for multi-class protein classification based on neural networks. The basic issue concerning the construction of neural network systems for protein classification is the sequence encoding scheme that must be used in order to feed the neural network. To deal with this problem we propose a method that maps a protein sequence into a numerical feature space using the matching scores of the sequence to groups of conserved patterns (called motifs) into protein families. We consider two alternative ways for identifying the motifs to be used for feature generation and provide a comparative evaluation of the two schemes. We also evaluate the impact of the incorporation of background features (2-grams) on the performance of the neural system. Experimental results on real datasets indicate that the proposed method is highly efficient and is superior to other well-known methods for protein classification.
\end{abstract}

Key words: protein sequence classification, neural networks, probabilistic motifs, MEME algorithm, motif-based features.

\section{INTRODUCTION}

$\mathbf{P}$ ROTEIN SEQUENCE ClASSIFICATION CONSTITUTES an important problem in biological sciences for annotating new protein sequences and detecting close evolutionary relationships among sequences. It deals with the assignment of sequences to known categories based on homology detection properties (sequence similarity). In several studies, protein classification has been examined at various levels, according to a top-down hierarchy in molecular taxonomy, consisting of superfamilies, families, and subfamilies (Dayhoff et al., 1978). Throughout this paper, we will use the terms family (or subfamily) and class interchangeably to denote any collection of sequences that are presumed to share common characteristics and belong to the same category.

Various approaches have been developed for solving the protein classification problem. Most of them are based on appropriately modeling protein families, either directly or indirectly. Direct modeling techniques use a training set of sequences to build a model that characterizes the family of interest. Hidden Markov models (HMMs) are a widely used probabilistic modeling method for protein families (Durbin et al., 1998) that provides a probabilistic measurement (score) of how well an unknown sequence fits to a family. Indirect techniques use direct models as a preprocessing tool in order to extract useful sequence features. In this way,

Department of Computer Science and Biomedical Research Institute - FORTH, University of Ioannina, GR-45110 Ioannina, Greece. 
sequences of variable length are transformed into fixed-length input vectors that are subsequently used for training discriminative models, such as neural networks.

In protein sequences, motifs or patterns enclose significant homologous attributes, since they correspond to conserved regions in protein families holding useful structural and functional biological properties. They can be considered as islands of amino acids conserved in the same order of a given family. Therefore, they can be seen as local features characterizing the sequences. Motifs can be either deterministic or probabilistic (Brāzma et al., 1998; Rigoutsos et al., 2000). Deterministic motifs follow grammatical inference properties in order to syntactically describe conserved regions of homologous sequences. The PROSITE database (Hofmann et al., 1999) represents a large collection of such motifs used to identify protein families. On the other hand, probabilistic motifs are more flexible models, and they provide a probabilistic matching score of a sequence to a motif. The BLOCKS database (Henikoff and Henikoff, 1994) is an example of ungapped probabilistic motifs. In any case, motif models are suitable for constructing efficient similarity score functions that can be subsequently used as local features for protein classification. An example is presented by Ma and Wang (2000), and by Wang et al. (2001) where motif-based local features are produced based on the minimum description length (MDL) principle for the case of deterministic motif models.

The background information also constitutes another source for extracting features from sequence data. The determination of the background features, also defined as global features, is usually made by using the 2 -gram encoding scheme that counts the occurrences of two consecutive amino acids in protein sequences (Wang et al., 2001). In the case of protein sequences (generated from the alphabet of the 20 aminoacids), there are 400 possible 2 -grams that produce a large feature space. A recent approach (Almeida and Vinga, 2002) proposes a scheme for globally encoding sequences, where each amino acid character is initially represented as a unique binary number with $n$ bits ( $n=5$ for the 20 aminoacids) and then each sequence is mapped into a position inside the $n$-dimensional hypercube.

In this paper, we focus on building efficient neural classifiers for discriminating multiple protein families by using appropriate local features that have been extracted by efficient probabilistic motif models. As motifs constitute family diagnostic signatures, our aim is to exploit this information by constructing a neural network scheme that exploits motif-based (local) features.

The proposed method can be considered as combining an unsupervised with a supervised learning technique. Starting by applying a motif-discovery (unsupervised) algorithm, we identify probabilistic motifs in a training set of multiclass sequences. This can be achieved in two alternative ways: applying the algorithm for motif discovery either to each family training set separately (class-dependent motifs), or to the whole dataset of training sequences (class-independent motifs). The discovered motifs are then used to convert each sequence to a numerical input vector that subsequently can be applied to a typical feedforward neural network. Using a Bayesian regularization training technique, the neural network parameters are adjusted, and therefore a classifier is obtained suitable for predicting the family of an unlabeled sequence.

The next section provides a brief overview of statistical and neural techniques proposed for classifying biological sequences, while Section 3 describes the proposed method. Experimental results obtained using several sets of protein families are presented in Section 4, along with a comparison with other known protein classification approaches. Finally, Section 5 summarizes the proposed classification scheme and specifies directions for future research.

\section{PROTEIN CLASSIFICATION METHODS}

One class of methods for protein sequence classification work directly with sequence information and establish classification criteria based on sequence homology properties. In the general scheme, a representative set of sequences is selected for each protein family and used to build an appropriate model for each family. The classification of an unknown sequence is made by selecting the family that best matches according to the model homology mechanism. This can be considered as a simple nearest neighbor scheme that ranks sequence similarities and selects the best ranking.

The popular BLAST tool (Altshul et al., 1990) represents the simplest nearest neighbor approach and exploits pairwise local alignments to measure sequence similarity. The BLAST technique compares protein 
queries with a protein database of labeled sequences and produces normalized alignment scores for each comparison by calculating the corresponding expectation values ( $E$-values). The classification procedure is based on the selection of the label of the sequence that produces the best pairwise alignment score (i.e., minimum $E$-value).

Another type of direct modeling methods is based on hidden Markov models (HMMs) (Durbin et al., 1998; Karplus et al., 1998). After constructing an HMM for each family, protein queries can be easily scored against all established HMMs by calculating the log-likelihood of each model for the unknown sequence and then selecting the class label of the most likely model.

The Motif Alignment and Search Tool (MAST) (Bailey and Gribskov, 1998) is based on the combination of multiple motif-based statistical score values. According to this scheme, groups of probabilistic motifs discovered by the MEME algorithm (Bailey and Elkan, 1994), are used to construct protein profiles for the families of interest. The MAST algorithm successively estimates the significance of the match of a query sequence to a family model as the product of the $p$-values of each motif match score. This measure (called $E$-value) can then be used to select the family of the unknown sequence.

Neural network schemes for protein classification consist of two stages: the encoding stage, where discriminative numerical features are computed for each training sequence, and the decision stage, where the created feature vectors are used as input vectors to a neural network classifier. Various encoding schemes have been proposed in the literature that produce numerical features in the encoding stage based on the calculation of background features (global sequence homology) and local features (locally conserved family information) embedded in motifs. In the decision stage, feed-forward neural networks have been used trained either through back-propagation (Wu et al., 1996) or using Bayesian regularization (Ma and Wang, 2000; Wang et al., 2001). These approaches are characterized by the enormous size of the extracted input vectors, the imbalance between global and local features (more emphasis on global features), and the need for large training sets (since the number of network inputs is very large). For example, in Ma and Wang (2000) and in Wang et al. (2001) only one feature was responsible for carrying local information, while all the others were produced by the 2-grams encoding scheme (background features).

Support vector machines (SVMs) (Vapnik, 1979) have been also applied to protein homology detection problems. Such an approach, which has been introduced by Logan et al. (2001), feeds probabilistic score values from all motifs available (nearly 10,000) in the BLOCKS database (Henikoff and Henikoff, 1994) into an SVM classifier. Obviously, this scheme uses only local features, but the dimensionality of the input space is extremely high. Another method has been proposed by Jaakkola et al. (2000) and by Karchin et al. (2002) that combines hidden Markov models (HMMs) and SVMs for detecting remote protein homologies. In particular, an HMM is first trained to model a protein family, and then the observed probabilities (in the log space) of each sequence with respect to each parameter of the HMM are calculated. The obtained gradient-log-probability vectors are applied to an SVM to identify the decision boundary between the family and the rest of the protein universe.

\section{THE PROPOSED METHOD}

This paper studies the problem of classifying a set of $N$ protein sequences $\left.\mathbf{S}=\left\{\mathcal{S}_{\rangle},\right\rangle=\infty, \ldots, \mathcal{N}\right\}$ into $K$ classes. The set $\mathbf{S}$ is a union of positive example datasets $\mathcal{S}_{\|}$from $K$ different classes, i.e., $\mathbf{S}=\left\{\mathcal{S}_{\infty} \cup \ldots \cup \mathcal{S}_{\mathcal{K}}\right\}$, and can be seen as a subset of the complete set of all possible sequences over the amino acid alphabet $\left(\mathbf{S} \subseteq \Sigma^{*}\right)$.

Figure 1 illustrates the architecture of the proposed protein classification scheme. It consists of a search tool (unsupervised learning) for discovering probabilistic motifs in a set of $K$ protein families, a feature vector generator that converts protein sequences into feature vectors, and a decision module (neural network) for assigning a protein family to each input sequence. The following subsections describe in detail the major building blocks of the proposed architecture.

\subsection{Using motifs for feature generation}

Consider a finite alphabet consisting of set of characters $\Sigma=\left\{\alpha_{1}, \ldots, \alpha_{\Omega}\right\}(\Omega=20$ for protein sequences). We can probabilistically model a contiguous (ungapped) motif $M_{j}$ of length $W_{j}$ using a 


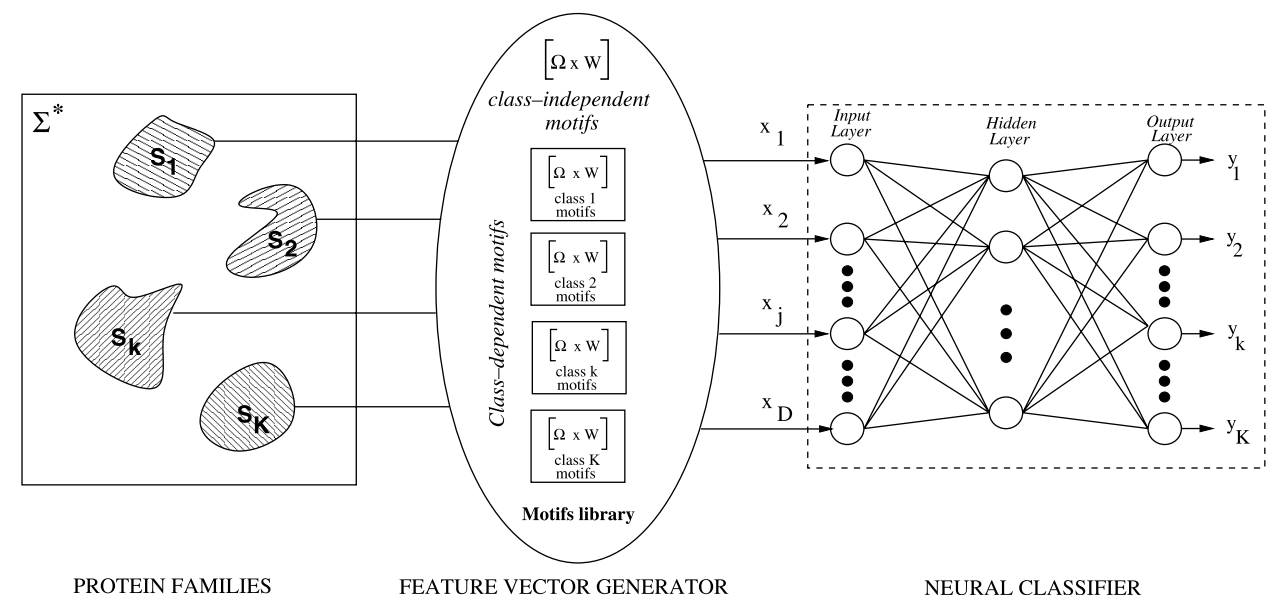

FIG. 1. The architecture of the proposed classification scheme.

position weight matrix $\left(P W M_{j}\right)$ that follows a multinomial character distribution. Each column $(l)$ of the matrix corresponds to a position $l$ in the motif sequence $\left(l=1, \ldots, W_{j}\right)$, where the column elements provide the probability of each character of the alphabet $p_{\alpha_{\xi}, l}(\xi=1, \ldots, \Omega)$ to appear in that position.

Let $s_{p}=a_{p, 1} \ldots a_{p, W_{j}}$ denote a segment of a sequence $S$ beginning at position $p$ and ending at position $p+W_{j}-1$. This represents a subsequence of length $W_{j}$. Totally, there are $L-W_{j}+1$ such subsequences for a sequence $S$ of length $L$. Then, we can define the probability that $s_{p}$ matches the motif $M_{j}$, or alternatively, has been generated by the model $P W M_{j}$ corresponding to that motif, using the following equation:

$$
P\left(s_{p} \mid M_{j}\right)=\prod_{l=1}^{W_{j}} p_{a_{p, l}, l} .
$$

A major advantage of using the probabilistic matrix $P W M_{j}$ is the ability to compute the corresponding position-specific score matrix $\left(P S S M_{j}\right)$ in order to score a sequence. The $P S S M_{j}$ is a log-odds matrix calculating the logarithmic ratio $r_{\alpha_{\xi}, l}$ of the probabilities $p_{\alpha_{\xi}, l}$ suggested by the $P W M_{j}$ and the corresponding general relative frequencies of aminoacids $\rho_{\alpha_{\xi}}$ in the family. ${ }^{1}$ According to the definition of $P S S M_{j}$, the score value $f_{j}\left(s_{p}\right)$ of a subsequence $s_{p}$ of a sequence $S$ can be defined as

$$
f_{j}\left(s_{p}\right)=\sum_{l=1}^{W_{j}} \log \left(\frac{p_{a_{p, l}, l}}{\rho_{a_{p, l}}}\right)=\sum_{l=1}^{W_{j}} r_{a_{p, l}, l} .
$$

At the sequence level, the score value of a protein sequence $S$ against a motif $M_{j}$ can be determined as the maximum value among all scores of the possible subsequences of $S$, i.e.,

$$
f_{j}(S)=\max _{1 \leq p \leq L-W_{j}+1} f_{j}\left(s_{p}\right) .
$$

It must be noted that it is possible to adopt other definitions for scoring a sequence, such as setting scores below a certain threshold equal to zero (Bailey and Gribskov, 1998).

If we assume that we have discovered a group of $D$ motifs in the set of sequences $\mathbf{S}$, we can generate a $D$-dimensional numerical feature space and map each sequence $S_{i}$ into a vector $\mathbf{x}_{i}$ in the $D$-dimensional feature space by calculating the score values $x_{i j}=f_{j}\left(S_{i}\right)(j=1, \ldots, D)$ for each of the $D$ motif models.

\footnotetext{
${ }^{1}$ The general relative frequencies of amino acids indicate the background information in a protein family and can be presented as a probabilistic vector $\rho$ of size $\Omega=20$.
} 


\subsection{Finding probabilistic motifs in protein sequences}

Several approaches have been proposed for discovering probabilistic motifs in a set of unaligned biological sequences. CONSENSUS (Hertz and Stormo, 1999), the Gibbs sampler (Lawrence et al., 1993), and MEME (Bailey and Elkan, 1994) are examples of such methods that identify multiple shared motifs in protein families. We have selected the MEME approach for the motif identification component of our strategy, since it has been widely used in biological applications and directly extracts position-specific score matrices. Below, we briefly describe this algorithm and propose two ways to integrate it in our classification system.

The MEME algorithm follows an iterative procedure, which applies at each iteration a two-component mixture model to discover one motif of length $W$. In the two-component model, one component describes the motif (ungapped common subsequences of length $W$ ) while the other component models the background information. Multiple motifs can be found by sequentially fitting the two-component model to the set of sequences that remain after removing the sequences containing occurrences of the already identified motifs.

In particular, MEME (Bailey and Elkan, 1994) uses the Expectation Maximization (EM) algorithm (Dempster et al., 1977) to maximize the log-likelihood function of the two-component mixture model, i.e., to estimate the elements of the corresponding position weight matrix. ${ }^{2}$ Furthermore, MEME provides a strategy for locating efficient initial parameter values in order to prevent the EM algorithm from getting stuck in local optima (Bailey and Elkan, 1994). The $D$ motif models $P W M_{j}(j=1, \ldots, D)$ discovered by MEME can be of either fixed or variable length $W_{j}$. In our experimental studies, both types of motifs will be examined to evaluate the impact of this decision on the performance of the neural classifier.

In order to discover a group of motifs from a multiclass training set of sequences (containing sequences of $K$ classes), two alternative approaches can be followed. The first approach is to apply the MEME algorithm $K$ times, separately to the training sequences of each protein family. Then, putting all the discovered $K$ family profiles together, we can form the final group of $D$ motifs. An alternative approach is to apply the motif-discovery algorithm only once to the total training set $\mathbf{S}$, ignoring class labels. In this way, we do not allow the algorithm to directly create $K$ protein family profiles, but rather to discover $D$ class-independent motifs.

The advantage of the second approach is the ability of taking into account local similarity measurements in the whole training set, without restricting the search procedure to a single class. Therefore, possible partial homologies among sequences from different families can be defined that may prove helpful for the classification task. On the other hand, a disadvantage of the class-independent approach is that the $D$ discovered motifs may not be equally distributed among the $K$ families. This may result in insufficient modeling of some families, thus leading to performance deterioration. During experiments, both motifdiscovery strategies will be considered and evaluated.

\subsection{Construction of a neural classifier}

After discovering $D$ motifs and constructing the $D$-dimensional feature space, the last stage in our methodology is to implement and train a feed-forward neural network that will be able to map the input vectors into the protein classes of interest. A typical network architecture is illustrated in Fig. 1. To construct the neural classifier, we use the training set $\mathbf{X}=\left\{\mathbf{x}_{i}, \mathbf{t}_{i}\right\}, i=1, \ldots, N$ consisting of positive examples $\mathbf{x}_{i}$ from the set of $K$ protein families. The target vector $\mathbf{t}_{i}$ is a binary vector of size $K$ indicating the class label of input $\mathbf{x}_{i}$; i.e., $t_{i k}=1$ if $\mathbf{x}_{i}$ corresponds to a sequence $S_{i}$ belonging to class $k$, and 0 otherwise. The output of the classifier is represented by the $K$-dimensional vector $\mathbf{y}_{i}$ where component $y_{i k}$ corresponds to class $k$. Based on this scheme, the predicted class $h\left(\mathbf{x}_{i}\right)$ of an unlabeled feature vector $\mathbf{x}_{i}$ corresponding to a query sequence $S_{i}$ is given by the index of the output node with the largest value $y_{i c}$; i.e.,

$$
h\left(\mathbf{x}_{i}\right)=c: y_{i c}=\max _{1 \leq k \leq K} y_{i k}
$$

\footnotetext{
${ }^{2}$ The model used in our experiments assumes that there are zero or more nonoverlapping occurrences of the motif in each sequence of the dataset. Alternative models that can be used are the exactly one-occurrence-per-sequence and the zero-or-one-occurrence-per-sequence models.
} 
Setting a threshold value $\theta(\in[0,1])$, we can restrict the classifiers' decision to only those input vectors whose maximum output value surpasses this threshold. In this case, we can write

$$
h\left(\mathbf{x}_{i}, \theta\right)=c: y_{i c}=\max _{1 \leq k \leq K} y_{i k} \wedge y_{i c} \geq \theta .
$$

Parameter $\theta$ can be used to specify the sensitivity of the classifier.

In order to train the neural network, we used the Gauss-Newton Bayesian Regularization (GNBR) learning algorithm (Foresse and Hagan, 1997). This algorithm applies Bayesian regularization and implements a Gauss-Newton approximation to the Hessian matrix of the objective function.

In the Bayesian regularization framework, the objective function is formulated as the weighted sum of two terms: the sum of the squared errors $\left(E_{X}\right)$ and the sum of squares of the network weights $\left(E_{W}\right)$. Using Bayes' rule, the posterior probability distribution for the weights $\mathbf{w}$ of the network given a training set $\mathbf{X}$ can be written as follows:

$$
P(\mathbf{w} \mid \mathbf{X})=\frac{P(\mathbf{X} \mid \mathbf{w}) P(\mathbf{w})}{P(\mathbf{X})} .
$$

By properly choosing the prior distribution $P(\mathbf{w})$ and the likelihood function $P(\mathbf{X} \mid \mathbf{w})$, we can obtain the following expression (Bishop, 1995; Foresse and Hagan, 1997) for the posterior distribution:

$$
P(\mathbf{w} \mid \mathbf{X})=\frac{1}{Z_{F}} \exp \left(-\beta E_{X}-\alpha E_{W}\right)=\frac{1}{Z_{F}} \exp (-F(\mathbf{w})),
$$

where the $Z_{F}$ corresponds to the normalizing factor that is independent of the weights.

Maximizing the above posterior distribution is equivalent to minimizing the regularized objective function $F(\mathbf{w})$ :

$$
F(\mathbf{w})=\frac{\beta}{2} \sum_{i=1}^{N_{X}}\left\{\mathbf{y}_{i}-\mathbf{t}_{i}\right\}^{2}+\frac{\alpha}{2} \sum_{j=1}^{N_{W}} w_{j}^{2},
$$

where $N_{X}$ and $N_{W}$ represent the number of input vectors and network parameters, respectively. In order to estimate the normalizing factor $Z_{F}$, a Gaussian approximation can be used for the posterior distribution (MacKay, 1992) as obtained by the Taylor expansion of function $F(\mathbf{w})$ around the minimum value of the posterior, $\mathbf{w}_{M P}$. This gives the following estimation (Bishop, 1995):

$$
Z_{F}^{*}(\alpha, \beta)=\exp \left(-F\left(\mathbf{w}_{M P}\right)\right)(2 \pi)^{N_{W} / 2}|\mathbf{H}|^{-1 / 2},
$$

where $\mathbf{H}$ corresponds to the Hessian matrix of the regularized objective function and, therefore, optimal values for parameters $\alpha$ and $\beta$ at the minimum point $\mathbf{w}_{M P}$ can be computed as follows:

$$
\hat{\alpha}=\frac{\gamma}{2 E_{W}\left(\mathbf{w}_{M P}\right)} \text { and } \hat{\beta}=\frac{\gamma N_{X}}{2 E_{X}\left(\mathbf{w}_{M P}\right)} .
$$

The quantity $\gamma$ represents the effective number of network parameters $\mathbf{w}$ and can be defined using the eigenvalues of $H^{-1}$ as $\gamma=N_{W}-2 \alpha \operatorname{TrH}^{-1}$. In cases where the number of effective parameters is equal to the actual ones $\left(\gamma \approx N_{W}\right)$, more hidden units must be added to the network. Furthermore, the GNBR algorithm follows a Gauss-Newton approximation method (Foresse and Hagan, 1997) for calculating the Hessian matrix of $F(\mathbf{w})$ at the minimum point $\mathbf{w}_{M P}$, using the Levenberg-Marquardt optimization algorithm (Bishop, 1995). It must be noted that in our experiments, the best results for the GNBR algorithm were obtained by scaling the network inputs in the range $[-1,1]$.

\section{EXPERIMENTAL RESULTS}

Several experiments were conducted to evaluate the proposed method. The classification accuracy was measured by counting the sensitivity and specificity rates. In all $K$-class classification problems, each 
Table 1. The Two Prosite Families Used in the Experimental Study

\begin{tabular}{ccccccc}
\hline & Problem: PROSITE $1(K=6)$ & & \multicolumn{2}{c}{ Problem: PROSITE 2 $(K=7)$} \\
\cline { 1 - 2 } $\begin{array}{c}\text { PROSITE } \\
\text { family }\end{array}$ & $\begin{array}{c}\text { Positive } \\
\text { data }\end{array}$ & $\begin{array}{c}\text { Training set } \\
\text { (avg length of seqs) }\end{array}$ & & $\begin{array}{c}\text { PROSITE } \\
\text { family }\end{array}$ & $\begin{array}{c}\text { Positive } \\
\text { data }\end{array}$ & $\begin{array}{c}\text { Training set } \\
\text { (avg length of seqs) }\end{array}$ \\
\hline PS00030 & 302 & $20(370)$ & & PS00070 & 129 & $15(558)$ \\
PS00038 & 289 & $20(359)$ & & PS00077 & 155 & $15(502)$ \\
PS00061 & 317 & $20(299)$ & & PS00118 & 168 & $15(127)$ \\
PS00198 & 300 & $20(284)$ & & PS00180 & 123 & $15(408)$ \\
PS00211 & 574 & $30(478)$ & & PS00215 & 123 & $15(321)$ \\
PS00301 & 386 & $20(517)$ & & PS00217 & 148 & $15(490)$ \\
& & & PS00338 & 173 & $15(212)$ \\
\hline
\end{tabular}

protein family $\mathcal{S}_{\|}(k=1, \ldots, K)$ was randomly partitioned into training and test sequences, with the training set being only a small percentage $(5-10 \%)$ of the family dataset. Using the training datasets, experiments have been carried out using the MEME algorithm to discover groups of motifs. Two cases were considered: in the first case, the MEME algorithm has been applied separately to each training set providing a group of $D_{k}=5$ class-dependent motifs for each family $\mathcal{S}_{\|}{ }^{3}$ In the second case, the MEME algorithm was applied only once to the total training dataset (ignoring the class labels) to provide a group of $D=5 \times K$ class-independent motifs.

In any case, the obtained final group of $D$ motifs were used to transform each sequence of the dataset into a dataset with numerical $D$-dimensional feature vectors, denoted $\mathbf{X}_{s}$ for the class-dependent case and $\mathbf{X}_{g}$ for the class-independent case. Furthermore, we also experimented with the effect of the length $W$ of the discovered motifs to the performance of the proposed classifier, by applying the MEME algorithm with either fixed or variable motif length. We selected $W=20$ for the first case and the range $[10,30]$ for the second case. In summary, we have considered four distinct cases considering the application of MEME: discovering either class-dependent or class-independent motifs with either fixed or variable motif length. Therefore, for each classification problem, four distinct neural classifiers will be constructed and tested.

To evaluate classification performance, ROC (receiver operating characteristic) analysis was used. More specifically, we used the $\mathrm{ROC}_{50}$ curve which is a plot of the sensitivity as a function of false positives for various decision threshold values until 50 false positives are found.

For our experimental study, three real datasets were selected. In particular we have used protein families from the PROSITE database (Hofmann et al., 1999), which is a large collection of protein families together with their characteristic (deterministic) motifs. Two datasets with $K=6$ (PROSITE 1) and $K=7$ (PROSITE 2) classes from the PROSITE database (Hofmann et al., 1999) were selected, summarized in Table 1. Moreover, experiments have also been conducted on a dataset of G-protein coupled receptors (GPCR) (Horn et al., 1998), that is, a superfamily of cell membrane proteins. The GPCR database is hierarchically classified into five major classes and their subfamilies (Horn et al., 1998). We studied the problem of classifying subfamilies within the class A, since it dominates the whole GPCR database. As indicated by Karchin et al. (2002), the difficulty of recognizing GPCR subfamilies arises from the fact that the classification of the subfamilies has been made based on chemical properties rather than sequence homology. Therefore, members from different subfamilies may share strong homology, thus making their discrimination hard. Among 15 subfamilies consisting of class A, seven of them have been selected in our experimental study described in Table 2. The remaining eight subfamilies are of very small size, and it is difficult to construct an effective system for their discrimination. Details of the three datasets (family/subfamily names and their protein ID's) used in our experiments are given in the appendix.

\subsection{Local versus global features}

In this series of experiments, we assessed the impact of using 2-grams (background features) on the performance of the proposed classification scheme. For a sequence $S_{i}$ with length $L_{i}$, we define the feature

\footnotetext{
${ }^{3}$ Experiments with a greater number of motifs did not yield better classification performance.
} 
Table 2. Seven Families from the GPCR Class A Used in THE Experimental StUdy

\begin{tabular}{lcc}
\hline \multicolumn{3}{c}{ Problem: GPCR $(K=7)$} \\
\hline $\begin{array}{l}\text { GPCR Class A } \\
\text { subfamily }\end{array}$ & $\begin{array}{c}\text { Positive } \\
\text { data }\end{array}$ & $\begin{array}{c}\text { Training set } \\
\text { (avg length of seqs) }\end{array}$ \\
\hline Amine & 306 & $20(485)$ \\
Peptide & 654 & $30(383)$ \\
Hormone & 43 & $10(378)$ \\
Rhodopsin & 270 & $20(358)$ \\
Olfactory & 325 & $20(317)$ \\
Prostanoid & 43 & $10(721)$ \\
Nucleotide-like & 58 & $10(348)$ \\
\hline
\end{tabular}

value $g_{i q}$ for each 2-gram $q$ with respect to this sequence as

$$
g_{i q}=\frac{\mathcal{N}\left(q \mid S_{i}\right)}{L_{i}-1},
$$

where $\mathcal{N}\left(q \mid S_{i}\right)$ denotes the number of occurrence of the 2-gram feature $q$ in the sequence $S_{i}$. Obviously, the above equation gives the relative frequency of a 2 -gram feature in a sequence. In a training set $\mathbf{S}=\left\{S_{1}, S_{2}, \ldots, S_{N}\right\}$ of $N$ sequences, we can ignore redundant 2 -grams and consider only the $N_{g}$ features $g_{i q}$ that correspond to the most frequently occurring 2-grams. We select the $N_{g} 2$-grams occurring in at least half of the training sequences and by computing the corresponding $g_{i q}\left(q=1, \ldots, N_{g}\right)$ values for each sequence $S_{i}$, we construct the corresponding feature vectors to be fed in the neural classifier.

Table 3 presents the dimensionality of the feature spaces obtained using 2-grams and motifs for each dataset used in the experiments. It must be noted that we can further reduce the dimensionality of the 2-gram feature vectors using standard dimension reduction techniques, such as principal component analysis (PCA).

To assess the impact of the several feature types on the performance of the classification system, we have considered five different datasets:

- $\mathbf{X}_{\mathbf{s}}: D$ motif-based features separately identified for each family (class-dependent),

- $\mathbf{X}_{\mathbf{g}}: D$ motif-based class-independent features,

- $\mathbf{X}_{\mathbf{s}} \cup \mathbf{G}$ : $D$ motif-based class-dependent features along with $N_{g}$ 2-gram features,

- $\mathbf{X}_{\mathbf{g}} \cup \mathbf{G}: D$ motif-based class-independent features, along with $N_{g} 2$-gram features

- G: $N_{g}$ 2-gram features.

The neural network architecture had one hidden layer of either 10 (for the cases $\mathbf{X}_{s}$ and $\mathbf{X}_{g}$ ) or 20 nodes (for the other three cases).

Figure 2 displays the $\mathrm{ROC}_{50}$ curves obtained after training the five neural classifiers in each of the three classification problems, respectively. For each problem, two different graphs are presented concerning

Table 3. The Number of the Extracted Motif-Based

$(D)$ and 2-Gram $\left(N_{g}\right)$ Features that Corresponds

TO Each Dataset

\begin{tabular}{lcc}
\hline Problem & $N_{g}$ & $D$ \\
2-gram features & motif-based features \\
\hline PROSITE 1 & 174 & $5 \times 6=30$ \\
PROSITE 2 & 285 & $5 \times 7=35$ \\
GPCR & 152 & $5 \times 7=35$ \\
\hline
\end{tabular}



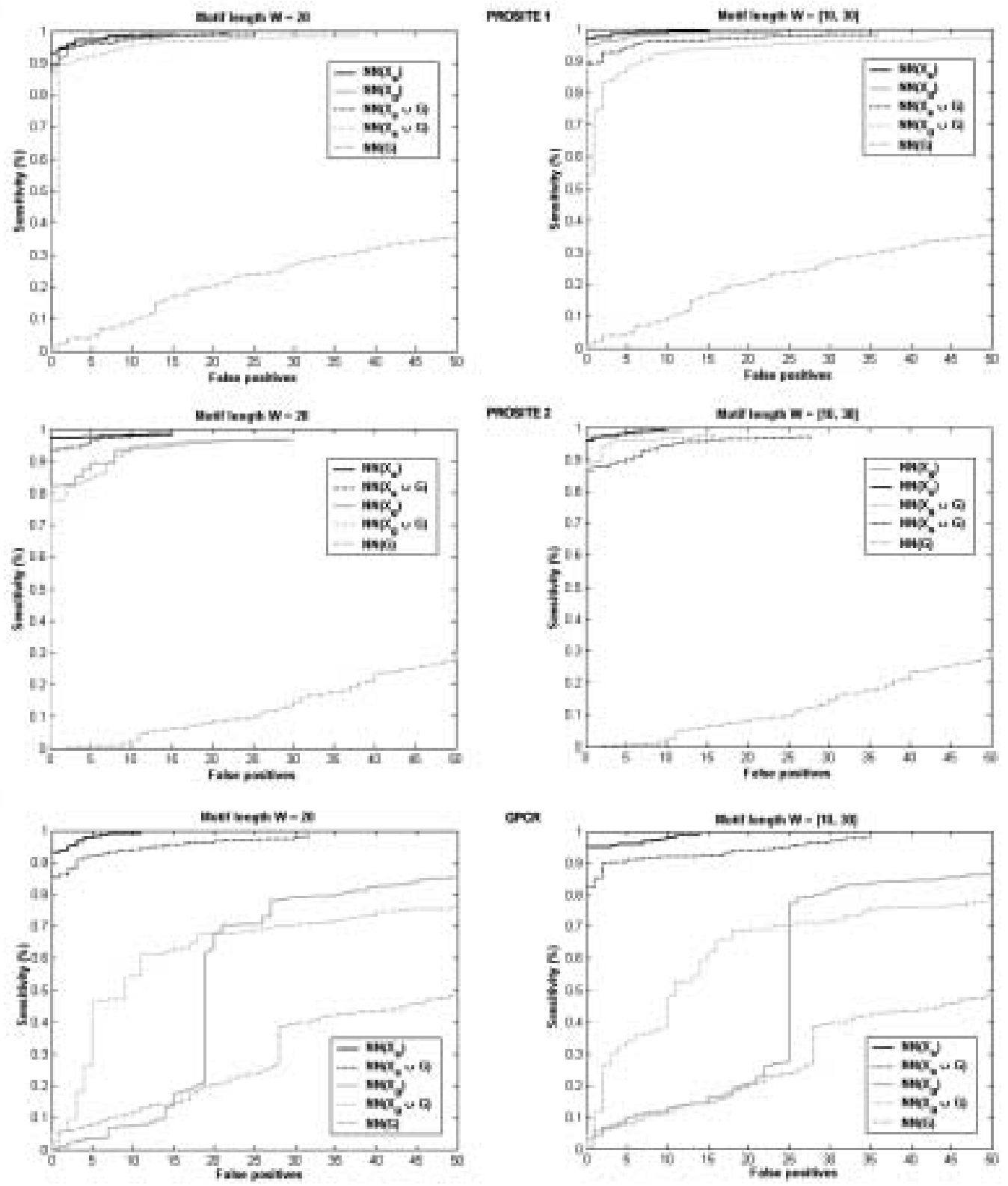

FIG. 2. ROC R0 $_{50}$ curves illustrating the performance of the neural classifier on the three datasets using the five different feature vectors.

motifs of fixed length $(W=20)$ and of variable length $W \in[10,30]$. Obviously, motif-based features themselves constitute an excellent source of information able to generate significant features and lead to the construction of efficient classifiers. In all cases, the neural networks trained by mixed features (e.g., $\left.\mathrm{NN}\left(\mathbf{X}_{s} \cup \mathbf{G}\right)\right)$ exhibit lower classification accuracy compared to the corresponding classifier trained with only motif-based features (e.g., $\mathrm{NN}\left(\mathbf{X}_{s}\right)$ ). Furthermore, the 2-grams features alone (case $\mathrm{NN}(\mathbf{G})$ ) do not seem to contain significant discriminant information.

Another observation that can be made from the $\mathrm{ROC}_{50}$ curves in Fig. 2 is related to the performance of the neural classifier with class-dependent motifs (network $\mathrm{NN}\left(\mathbf{X}_{s}\right)$ ) compared to that obtained with classindependent motifs (network $\mathrm{NN}\left(\mathbf{X}_{g}\right)$ ). In almost all cases, we obtained better classification results with the network $\mathrm{NN}\left(\mathbf{X}_{s}\right)$. One explanation for this behavior is that, when searching for a specific number $D$ of motifs in the whole training set (ignoring class labels), the algorithm may focus on some of the families 

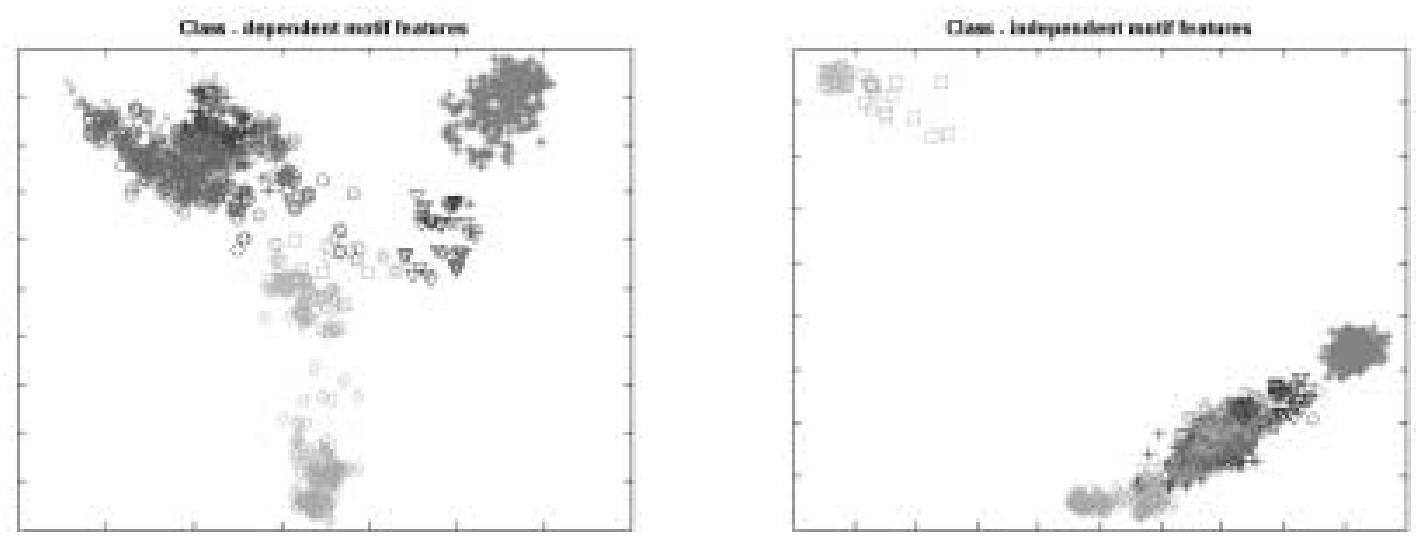

FIG. 3. The seven class regions in the GPCR dataset in the case of class-dependent and class-independent features. The data have been projected in two dimensions using PCA.

and leave the other families explored only partially. This possibly affects the satisfactory modeling of some families, since the discovered class-independent motifs may not be sufficient for describing them (only a few individual motifs are dedicated to this family). Experiments in the $\mathbf{X}_{g}$ datasets with MEME have shown that the allocation of motifs in most cases was not equal for all the $K$ families.

An example is shown in Fig. 3 that illustrates the constructed feature space of the $\mathbf{X}_{s}$ and $\mathbf{X}_{g}$ datasets in the case of the GPCR problem (seven classes), after projecting the 35-dimensional numerical to a twodimensional space using PCA. It can be observed that in the case of class-dependent motifs the protein classes exhibit less overlap while in the reduced feature space of class-independent motifs there is a significant overlapping among class regions, thus making the discrimination harder. A selection of higher values of $D$ probably would lead to better results for the class-independent case, but would simultaneously result in larger feature spaces or to the overestimation of some families.

\subsection{Comparison with other approaches}

We have also compared the neural classifier (with class-dependent motif-based features) with two other protein classification methods, namely, the MAST homology detection algorithm (Bailey and Gribskov, 1998) and the profile HMMs built using SAM, (Hughey and Krogh, 1996). In both MAST and SAM, each protein family (or subfamily) is transformed (indirectly or directly) into a probabilistic model-profile, and the test sequences are classified using the class of the profile with the best score value.

More specifically, the MAST procedure (Bailey and Gribskov, 1998) initially uses the MEME algorithm to discover groups of motifs separately for each one of the $K$ protein families. For each sequence in the testing set, the MAST algorithm combines the calculated $p$-values and estimates the significance of the observed match (called $E$-value) of the sequence to each of the $K$ groups of motifs. ${ }^{4}$ Then the query sequence is assigned to the class with the minimum $E$-value. The SAM method (Hughey and Krogh, 1996) works in a similar way by building an HMM for each one of the $K$ protein families (or subfamilies) instead of discovering groups of motifs. ${ }^{5}$

Figure 4 provides comparative results from the application of the proposed neural classifier, MAST and SAM, to the three datasets. We have created five ROC curves for each method (number of false positives versus sensitivity for several threshold values) until 25 false positives were found $\left(\mathrm{ROC}_{25}\right)$. The performance of the neural classifier and MAST was given by two curves respectively ${ }^{6}$ concerning motifs of fixed $(W=20)$ and variable length $(W=[10,30])$, while the last one corresponds to SAM performance.

\footnotetext{
${ }^{4}$ We use the meme and mast commands from the available MEME package v.3.0.4.

${ }^{5}$ We use the buildmodel and hmmscore commands from the available SAM package v.3.3.1.

${ }^{6}$ The curves for the neural classifier performance were the best plots from the corresponding $\mathrm{ROC}_{50}$ diagrams in
} Fig. 2. 

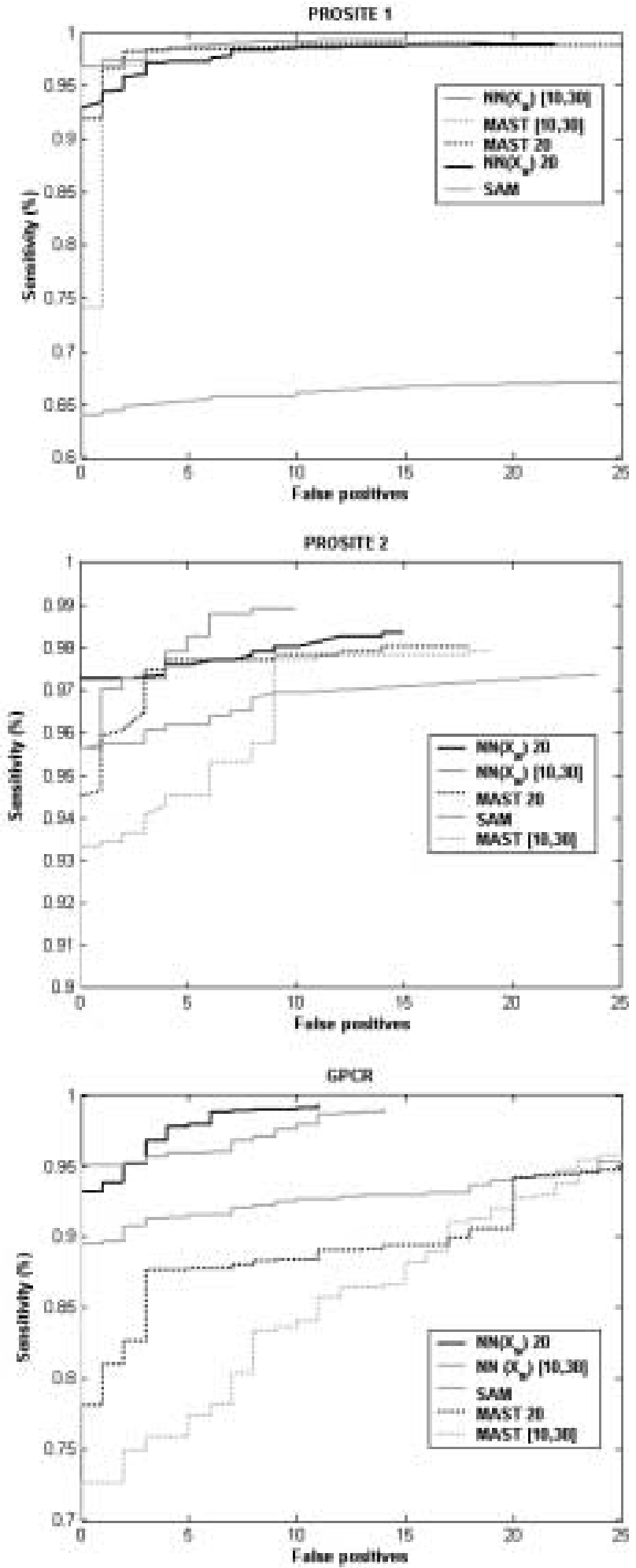

FIG. 4. ROC 25 curves for the three methods (neural (NN), MAST, and SAM) on the three datasets. 
In the case of MAST and SAM methods, ROC curves were obtained by setting several $E$-value thresholds. When the lowest estimated $E$-value for a query sequence was greater than the threshold, then the test sequence was considered unclassified.

The superior classification of the proposed neural approach is obvious from the plotted curves in all problems, offering greater sensitivity rates with perfect specificity (zero false positives). For the GPCR dataset, which is more difficult to discriminate, the classification improvement is more clear: a sensitivity rate of $99.30 \%$ was measured with only 11 false positives, while the corresponding results for MAST and SAM are $(95.76 \%, 25)$ and $(95.38 \%, 25)$, respectively. It is also important to stress the higher accuracy that the neural scheme achieves compared with MAST (dot lines). Although these two methods use the same groups of motifs, our method seems to offer a more efficient scheme for combining the motif match scores compared to the combination of their $p$-values as suggested by MAST. In addition, the neural classifier achieves fewer false positives with higher sensitivity rates in all datasets concerning either fixed or variable motif length. Again, the improvement is more clear in the plots corresponding to the GPCR dataset.

Regarding more carefully the three selected datasets, they can be considered as three different types of protein sequence classification problems. In particular, the PROSITE 1 dataset consists of diverse protein families in the sense that their corresponding PROSITE motifs are not very specific (such as in the case of PS00030 and PS00198) and they can be found in sequences from a large number of protein families. Hence, this application can be seen as a diverse protein family recognition problem. On the other hand, the PROSITE 2 dataset consists of protein families with more specific PROSITE motifs that can be distinguished more easily. Finally, the third dataset, GPCR, is related to the recognition of protein subfamilies within a broader protein family domain sharing strong homology.

In all the above three types of protein sequences classification problems, our approach has shown a superior classification performance providing better results in comparison with the two other approaches. As illustrated in Fig. 4, the SAM method seems to be unsuccessful in recognizing diverse protein families (PROSITE 1 case), and the obtained classification rate was low (the individual classification error for each diverse family was about 50\%). On the other hand, the performance of the MAST method was lower in the case of the GPCR subfamily recognition problem where sequences from different subfamilies share strong homology. Finally, in the case of recognizing simple protein families (PROSITE 2 dataset), all the three approaches provide similar classification rates, with the proposed neural scheme offering slightly better results.

\section{CONCLUSIONS}

In this paper, we have presented a neural network approach for the classification of protein sequences. The proposed methodology is motivated by the principle that in biological sequence analysis motifs can provide major diagnostic features for determining the class label of the unknown sequences. The method is implemented in two steps, where a preprocessing step (based on the MEME algorithm) is initially applied for discovering a group of probabilistic motifs appearing in the sequences. We have suggested and evaluated two alternative ways for motif discovery in a set of $K$-class sequences depending on whether the class labels are taken into account. Using the discovered motifs, a numerical feature vector is generated for each sequence by computing the matching score of the sequence to each motif. At the second stage of the proposed method, the extracted feature vectors are used as inputs to a feed-forward neural network trained using the Gauss-Newton Bayesian Regularization algorithm that provides the class label of a sequence.

Experiments were conducted on real datasets (using very small training sets), and comparisons were made with the MAST and SAM probabilistic methods. ROC curves were used as a performance indicator, and the experimental results clearly illustrate the superiority of the proposed neural system. In addition we have shown that background features do not constitute a useful source of information for the classification task since they do not lead to performance improvement.

In future work, more extensive experiments could be conducted to assess the performance of the method on specific protein superfamilies of important biological functions, as was the case with the GPCR dataset. Also, alternative methods could be implemented and tested, both in the classification stage (mixture models, SVMs, etc.) and in the motif discovery stage. 


\section{APPENDIX: DATASETS}

In the next tables proteins with bold ID's correspond to the training examples and the rest of them to the test set.

Table 4. Description of the PROSite 1 Dataset

\begin{tabular}{|c|c|}
\hline Family & Protein ID's \\
\hline PS00030 & 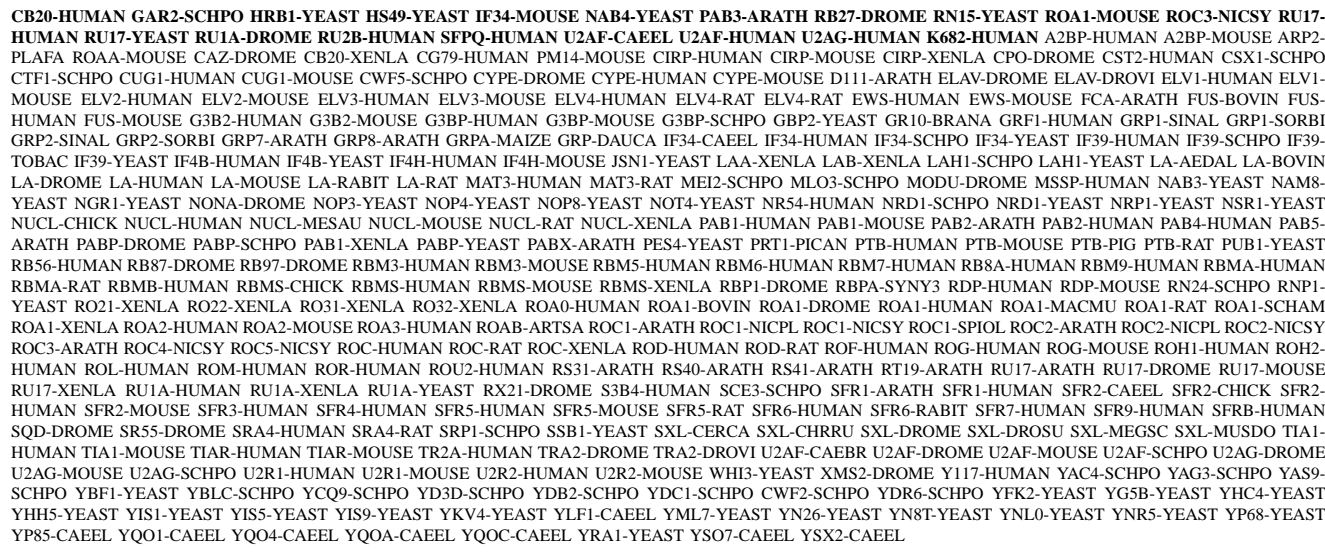 \\
\hline PS00038 & 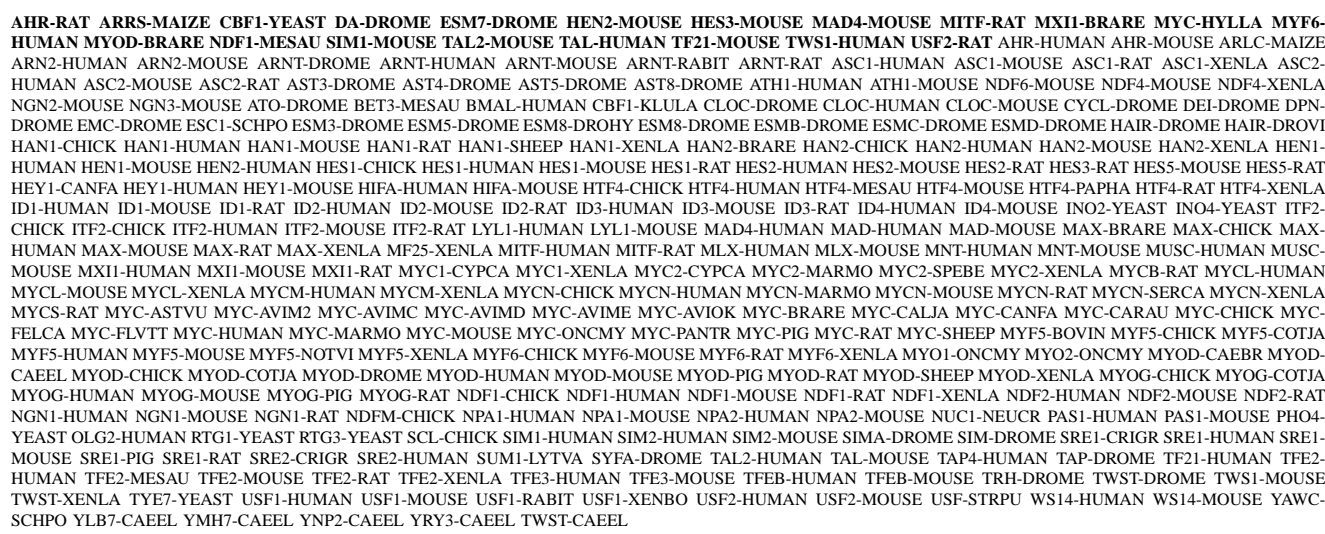 \\
\hline PS00061 & $\begin{array}{l}\text { 2BHD-STREX ADH2-DROMN ADH-DROMA ADH-DROMM ADH-DROSL DECR-RAT } \\
\text { STRVN } 1 \text { DNTA-ECOLI }\end{array}$ \\
\hline
\end{tabular}


TABLE 4. (Continued)

\begin{tabular}{|c|c|}
\hline Family & Protein ID's \\
\hline PS00198 & 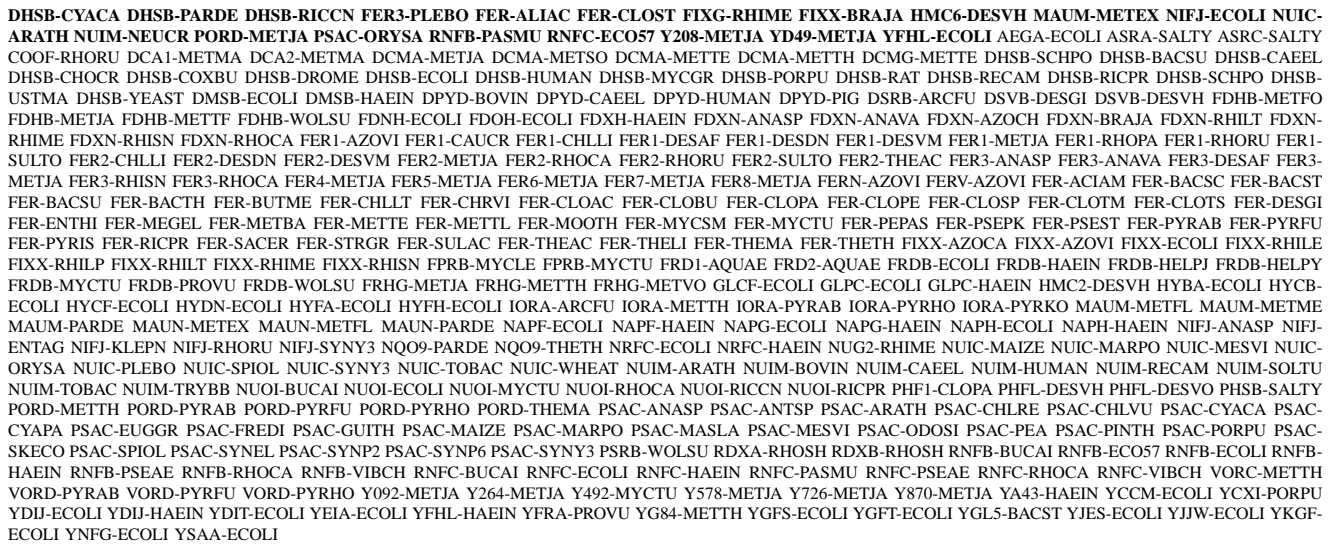 \\
\hline PS00211 & 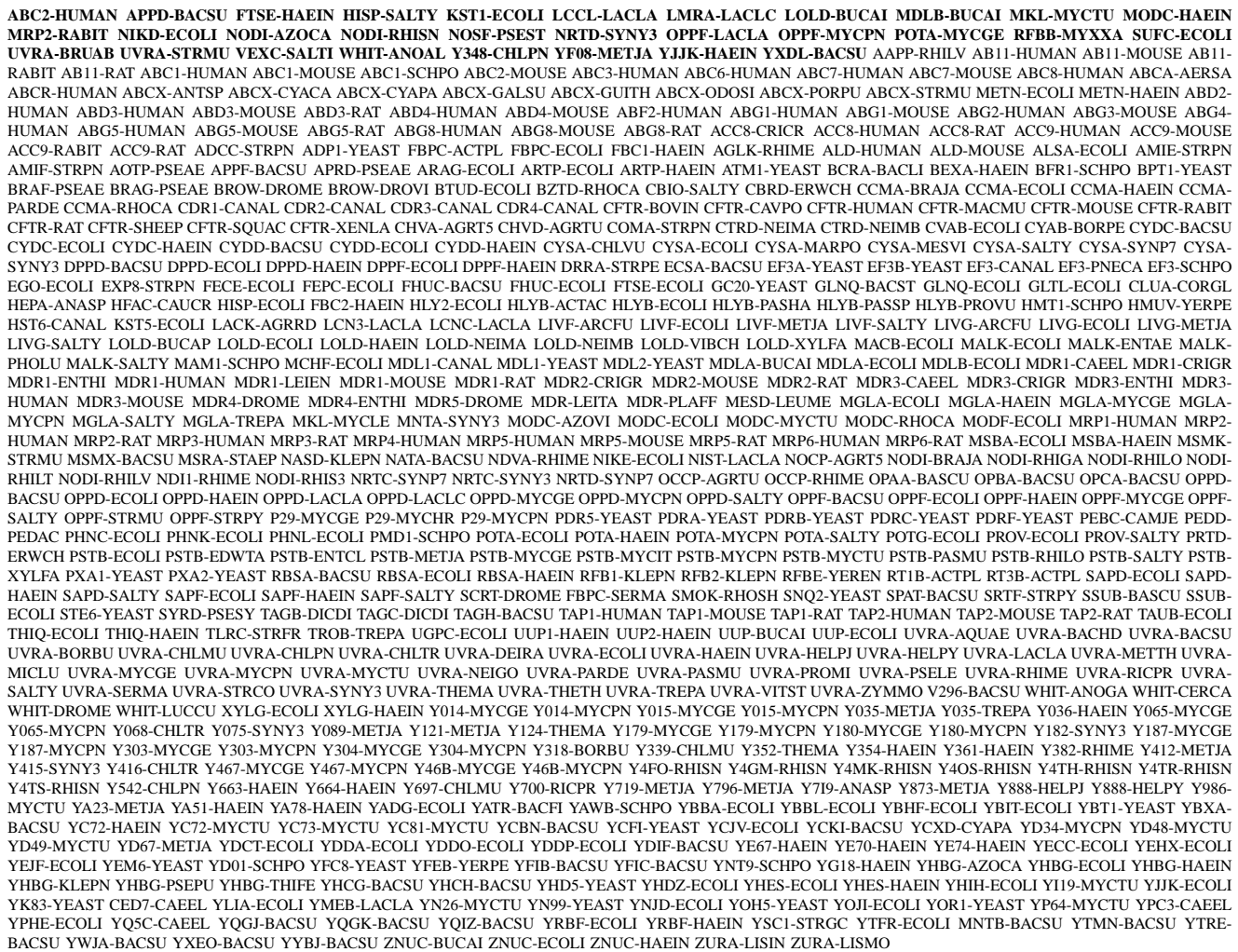 \\
\hline
\end{tabular}


TABle 4. (Continued)

\begin{tabular}{|c|c|}
\hline Family & Protein ID's \\
\hline PS00301 & 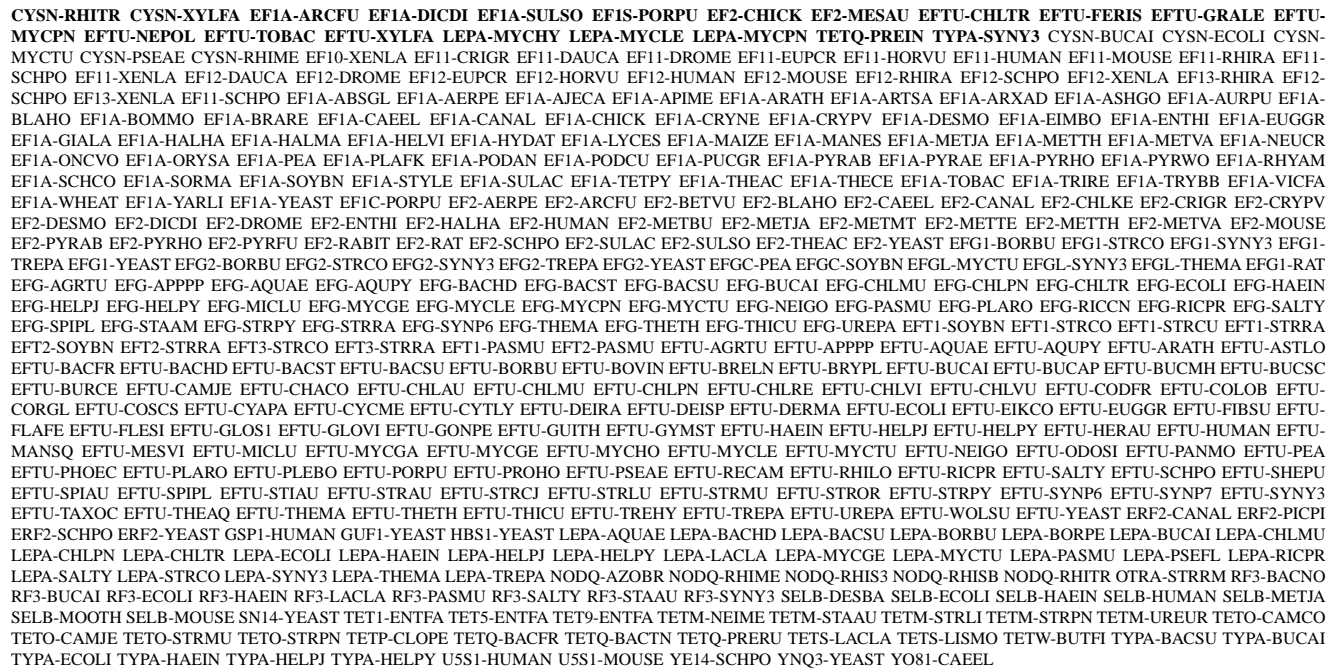 \\
\hline
\end{tabular}

Table 5. Description of the PROSite 2 Dataset

\begin{tabular}{|c|c|}
\hline Family & Protein ID's \\
\hline PS00070 & 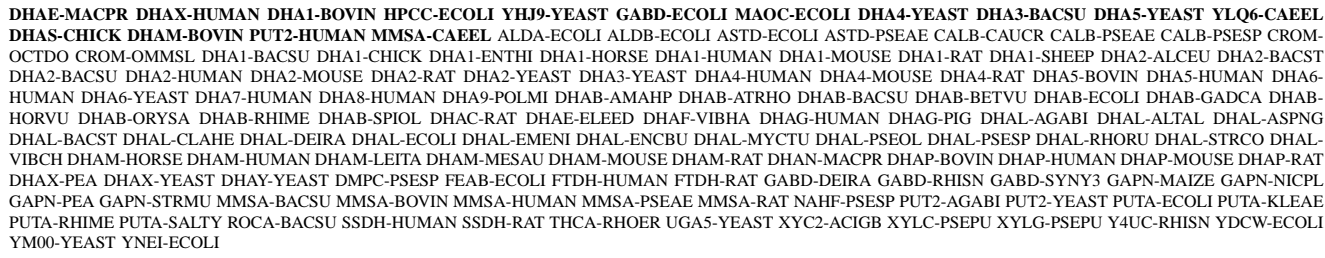 \\
\hline PS00077 & 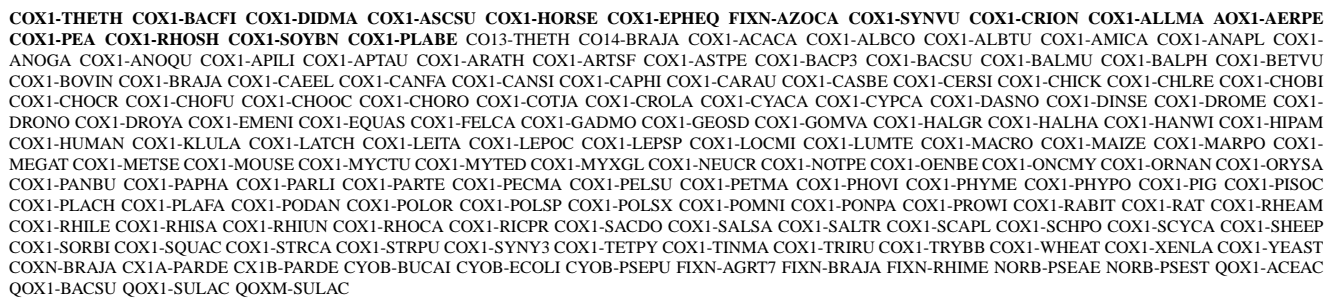 \\
\hline PS00118 & 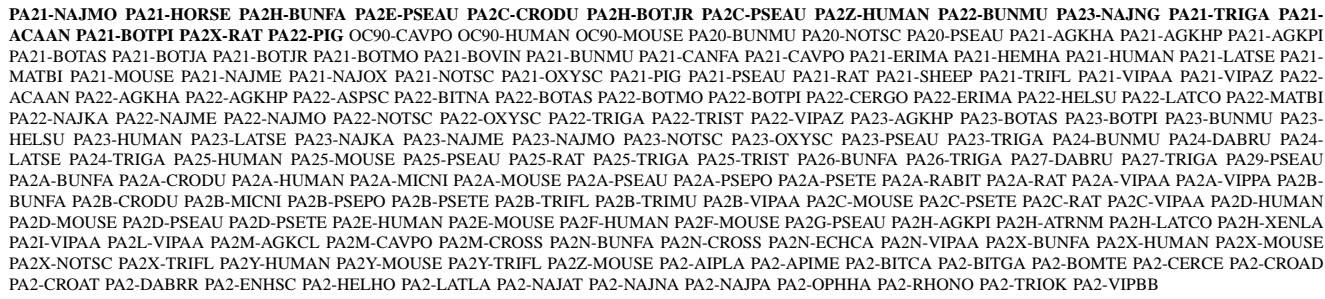 \\
\hline PS00180 & $\begin{array}{l}\text { GLNA-COLGL GLN4-PEA GLN2-DROME GLNA-HELPY GLNA-PANAR GLN3-RHILP GLN1-ARATH GLN5-MAIZE GLNA-PIG GLNA-PYRHO GLNA-THIFE GLNA- } \\
\text { SALTY GLN3-PHAVU GLNA-NICPL GLN2-DAUCA GLN1-ALNGL GLN1-BRAJA GLN1-CHLRE GLN1-DAUCA GLN1-DROME GLN1-FRAAL GLN1-LOTJA GLN1-MAIZE } \\
\text { GLN1-MEDSA GLN1-MYCTU GLN1-ORYYA GLN1-PEA GLN1-PHAVU GLN1-RHILV GLN1-RHIME GLN1-SOYBN GLN1-STRRP GLN1-STRVR GLN1-VITVI GLN2-ARATH } \\
\text { GLN2-BRAJA GLN2-CHLRE GLN2-FRAAL GLN2-HORVU GLN2-MAIZE GLN2-MEDSA GLN2-MYCTU GLN2-ORYSA GLN2-PEA GLN2-PHAVU GLN2-RHILP GLN2-RHIME } \\
\text { GLN2-SOYBN GLN2-STRHY GLN2-STRVR GLN2-VITVI GLN3-HORVU GLN3-LUPAN GLN3-MAIZE GLN3-MEDSA GLN3-ORYSA GLN3-PEA GLN3-RHIME GLN4-MAIZE } \\
\text { GLN4-PHAVU GLNA-AGABI GLNA-ANASP GLNA-AQUAE GLNA-ARCFU GLNA-AZOBR GLNA-AZOCA GLNA-AZOVI GLNA-BACCE GLNA-BACFR GLNA-BACSU GLNA- } \\
\text { BOVIN GLNA-BUTFI GLNA-CAEEL GLNA-CHICK GLNA-CLOSA GLNA-CRILO GLNA-DUNSA GLNA-ECOLI GLNA-FREDI GLNA-HAEIN GLNA-HALN1 GLNA-HALVO } \\
\text { GLNA-HELPI GLNA-HUMAN GLNA-LACDE GLNA-LACLA GLNA-LACSA GLNA-LUPLU GLNA-METCA GLNA-METJA GLNA-METMP GLNA-METTH GLNA-METVO } \\
\text { GLNA-MOUSE GLNA-NEIGO GLNA-PASMU GLNA-PINSY GLNA-PROVU GLNA-PYRAB GLNA-PYRFU GLNA-PYRKO GLNA-PYRWO GLNA-RAT GLNA-RHOCA GLNA- } \\
\text { RHOSH GLNA-SCHPO GLNA-SQUAC GLNA-STAAU GLNA-STRCO GLNA-SULAC GLNA-SULSO GLNA-SYNP2 GLNA-SYNY3 GLNA-THEMA GLNA-TRITH GLNA-VIBAL } \\
\text { GLNA-VIBCH GLNA-VIGAC GLNA-XENLA GLNA-YEAST GLNC-BRANA GLNC-MAIZE YCJK-ECOLI }\end{array}$ \\
\hline
\end{tabular}


Table 5. (Continued)

\begin{tabular}{|c|c|}
\hline Family & Protein ID's \\
\hline PS00215 & $\begin{array}{l}\text { UCP5-HUMAN AR13-NEUCR SA18-MOUSE YIA6-YEAST SHM1-YEAST ADT1-BOVIN ADT2-WHEAT UCP3-BOVIN M2OM-RAT YAD8-SCHPO UCP1-MOUSE TXTP- } \\
\text { HUMAN DNC-HUMAN ADT3-YEAST ADT3-HUMAN ADT1-ARATH ADT1-GOSHI ADT1-HUMAN ADT1-MAIZE ADT1-MOUSE ADT1-RAT ADT1-SOLTU ADT1-WHEAT } \\
\text { ADT1-YEAST ADT2-ARATH ADT2-HUMAN ADT2-MAIZE ADT2-MOUSE ADT2-RAT ADT2-SOLTU ADT2-YEAST ADT3-BOVIN ADT-ANOGA ADT-CHLKE ADT-CHLRE } \\
\text { ADT-DROME ADT-KLULA ADT-NEUCR ADT-ORYSA ADT-SCHPO BT1-MAIZE CG69-HUMAN CMC1-CAEEL CMC1-DROME CMC1-HUMAN CMC1-YEAST CMC2-CAEEL } \\
\text { CMC2-HUMAN CMC2-MOUSE CMC3-CAEEL DIC-HUMAN DIC-MOUSE ECHP-MOUSE FLX1-YEAST GDC-BOVIN GDC-HUMAN GDC-RAT LEU5-YEAST M2OM-BOVIN } \\
\text { M2OM-HUMAN M2OM-MOUSE MCAT-HUMAN MCAT-RAT MFT-HUMAN MPCP-BOVIN MPCP-CAEEL MPCP-CHOFU MPCP-HUMAN MPCP-RAT MPCP-YEAST MRS3- } \\
\text { YEAST MRS4-YEAST ODC1-YEAST ODC2-YEAST ODC-HUMAN ORT1-HUMAN ORT1-MOUSE ORT1-YEAST ORT2-HUMAN P47A-CANBO P47B-CANBO PET8-YEAST } \\
\text { PM34-HUMAN PM34-MOUSE PMT-YEAST RIM2-YEAST SA18-HUMAN SFC1-YEAST TXTP-BOVIN TXTP-CAEEL TXTP-RAT TXTP-YEAST UCP1-BOVIN UCP1-HUMAN } \\
\text { UCP1-MESAU UCP1-RABIT UCP1-RAT UCP2-BRRARE UCP2-CANFA UCP2-CYPCA UCP2-HUMAN UCP2-MOUSE UCP2-PIG UCP2-RAT UCP3-CANFA UCP3-HUMAN UCP3- } \\
\text { MOUSE UCP3-PIG UCP3-RAT UCP4-HUMAN UCP5-MOUSE YD1K-SCHPO YDE9-SCHPO YE08-SCHPO YEA6-YEAST YEO3-YEAST YFL5-YEAST YG2O-YEAST YG5F- } \\
\text { YEAST YM39-YEAST YMC1-YEAST YMC2-YEAST YQ51-CAEEL }\end{array}$ \\
\hline PS00217 & $\begin{array}{l}\text { GTR1-RAT IOLF-BACSU CSBC-BACSU GTR5-HUMAN KHT2-KLULA PH84-YEAST NANT-ECOLI GHT3-SCHPO HUP1-CHLKE HGT1-CANAL GTR4-RAT GTR1- } \\
\text { CHICK MMLH-ALCEU OUSA-ERWCH PHDK-NOCSK AGT1-YEAST ARAE-BACSU ARAE-ECOLI ARAE-KLEOX BENK-ACICA CIT1-ECOLI CT11-KLEPN CIT1-SALTY } \\
\text { GAL2-YEAST GALP-ECOLI GHT2-SCHPO GHT4-SCHPO GHT5-SCHPO GHT6-SCHPO GIT1-YEAST GLCP-SYNY3 GLF-ZYMMO GT10-HUMAN GT11-HUMAN GTR1-BOVIN } \\
\text { GTR1-HUMAN GTR1-LEIDO GTR1-MOUSE GTR1-PIG GTR1-RABIT GTR1-SHEEP GTR2-BOVIN GTR2-CHICK GTR2-HUMAN GTR2-LEIDO GTR2-MOUSE GTR2-PIG GTR2- } \\
\text { RAT GTR3-BOVIN GTR3-CANFA GTR3-CHICK GTR3-DROME GTR3-HUMAN GTR3-MOUSE GTR3-PIG GTR3-RABIT GTR3-RAT GTR3-SHEEP GTR4-BOVIN GTR4-CANFA } \\
\text { GTR4-HUMAN GTR4-MOUSE GTR4-PIG GTR5-BOVIN GTR5-MOUSE GTR5-RABIT GTR5-RAT GTR6-HUMAN GTR8-BOVIN GTR8-HUMAN GTR8-MOUSE GTR8-RAT GTR9- } \\
\text { HUMAN HEX6-RICCO HGT1-KLULA HUP2-CHLKE HUP3-CHLKE HXT0-YEAST HXT1-YEAST HXT2-YEAST HXT3-YEAST HXT4-YEAST HXT5-YEAST HXT6-YEAST } \\
\text { HXT7-YEAST HXT8-YEAST HXT9-YEAST HXTA-YEAST HXTC-YEAST HXTD-YEAST HXTE-YEAST HXTF-YEAST HXTG-YEAST ITR1-SCHPO ITR1-YEAST ITR2-SCHPO } \\
\text { ITR2-YEAST JEN1-YEAST KGTP-ECOLI LACP-KLUULA MA3T-YEAST MA6T-YEAST MAXT-YEAST MHPT-ECOLI MUCC-ACICA MYCT-HUMAN PCAK-ACICA PCAK- } \\
\text { PSEPU PRO1-LEIEN PROP-ECOLI PROP-SALTY QAY-NEUCR QUTD-EMENI RAG1-KLULA RCO3-NEUCR RGT2-YEAST SHA-ECOLI SNF3-YEAST STA-RICCO STC-RICCO } \\
\text { STL1-YEAST STP1-ARATH STP-SPIOL TH11-TRYBB TH12-TRYBB TH23-TRYBB TH2A-TRYBB XYLE-ECOLI XYLT-LACBR Y281-HAEIN Y418-HAEIN YAAU-ECOLI YAEC- } \\
\text { SCHPO YB04-HAEIN YB91-YEAST YCEI-BACSU YDFJ-ECOLI YDJE-ECOLI YDJK-ECOLI YFE0-YEAST YFIG-BACSU YGCS-ECOLI YGK4-YEAST YHJE-ECOLI YIR0- } \\
\text { YEAST YJHB-ECOLI YOU1-CAEEL YYAJ-BACSU }\end{array}$ \\
\hline PS00338 & $\begin{array}{l}\text { SOMA-TRIVU PRL-CHICK PRL-PAROL SOMA-MACMU PRL-MOUSE SOMA-ACALA PLL2-MESAU SOML-SIGGU SOMA-ESOLU SOM2-CARAU SOMA-CANFA } \\
\text { PRL-SHEEP SOM2-HUMAN PRL-HORSE SOMA-PANTR GHR1-RAT GHR3-RAT GHR4-RAT PLF1-MOUSE PLF2-MOUSE PLF3-MOUSE PLFR-MOUSE PLL1-BOVIN PLL1- } \\
\text { MOUSE PLL1-RAT PLL2-BOVIN PLL2-MOUSE PLL2-RAT PLLV-RAT PLL-HUMAN PLL-SHEEP PRL1-ALLMI PRL1-CRONO PRL1-ONCKE PRL1-OREMO PRL2-ALLMI PRL2- } \\
\text { CRONO PRL2-ONCKE PRL2-ONCTS PRL2-OREMO PRL-ANGAN PRL-BALBO PRL-BOVIN PRL-BUFJA PRL-CAMDR PRL-CAPHI PRL-CARAU PRL-CHEMY PRL-CORAU } \\
\text { PRL-CYPCA PRL-DICLA PRL-FELCA PRL-HUMAN PRL-HYPMO PRL-HYPNO PRL-ICTPU PRL-LOXAF PRL-MACMU PRL-MELGA PRL-MESAU PRL-MONDO PRL-MUSVI } \\
\text { PRL-ONCMY PRL-PIG PRL-PROAT PRL-RABIT PRL-RAT PRL-SALSA PRL-SPAAU PRL-TRIVU PRR1-BOVIN PRR2-BOVIN PRR3-BOVIN PRR4-BOVIN PRRA-RAT PRRB- } \\
\text { RAT PRRC-RAT SOM1-ACIGU SOM1-CARAU SOMA-ONCKE SOM1-ONCNE SOM1-SPAAU SOM2-ACIGU SOM2-MACMU SOM2-ONCMY SOM2-ONCNE SOM2-PANTR } \\
\text { SOM2-SPAAU SOMA-ACABU SOMA-ANAPL SOMA-ANGJA SOMA-BALBO SOMA-BOVIN SOMA-BUBBU SOMA-BUFMA SOMA-CALJA SOMA-CARDE SOMA-CEREL } \\
\text { SOMA-CHEMY SOMA-CHICK SOMA-CORAU SOMA-CORLV SOMA-CRONO SOMA-CTEID SOMA-CYPCA SOMA-DICLA SOMA-FELCA SOMA-FUGRU SOMA-GALSE } \\
\text { SOMA-HETFO SOMA-HORSE SOMA-HUMAN SOMA-ICTPU SOMA-KATPE SOMA-LABRO SOMA-LAMPA SOMA-LATCA SOMA-LEPOS SOMA-LOXAF SOMA-MELGA } \\
\text { SOMA-MESAU SOMA-MISMI SOMA-MONDO SOMA-MORSA SOMA-MOUSE SOMA-MUSVI SOMA-NYCPY SOMA-ODOAR SOMA-ONCKE SOMA-ONCKI SOMA-ONCMA } \\
\text { SOMA-ONCTS SOMA-OREMO SOMA-ORENI SOMA-PAGMA SOMA-PANPG SOMA-PAROL SOMA-PERFV SOMA-PIG SOMA-PRIGL SOMA-PROAN SOMA-PSECR SOMA- } \\
\text { RABIT SOMA-RANCA SOMA-RAT SOMA-SAIBB SOMA-SALSA SOMA-SCIOC SOMA-SEBSC SOMA-SERQU SOMA-SHEEP SOMA-SIGGU SOMA-SOLSE SOMA-SPAAU } \\
\text { SOMA-STRCA SOMA-THUAL SOMA-THUTH SOMA-TRITC SOMA-VERVA SOMA-VULVU SOMA-XENLA SOMB-XENLA SOML-ACITR SOML-ANGAN SOML-CARAU } \\
\text { SOML-CYCLU SOML-GADMO SOML-HIPHI SOML-ICTPU SOML-ONCKE SOML-PAROL SOML-PROAN SOML-SCIOC SOML-SOLSE SOML-TETMU }\end{array}$ \\
\hline
\end{tabular}

TABle 6. Description of the GPCR Dataset

\begin{tabular}{|c|c|}
\hline Subfamily & Protein ID's \\
\hline Amine & 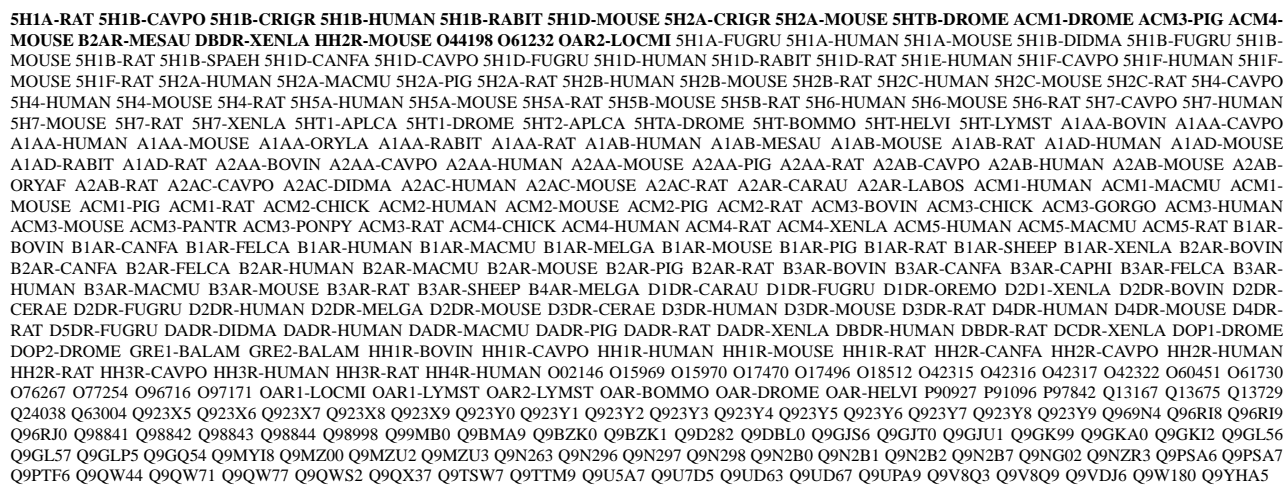 \\
\hline
\end{tabular}


Table 6. (Continued)

\begin{tabular}{|c|c|}
\hline Subfamily & Protein ID's \\
\hline Peptide & 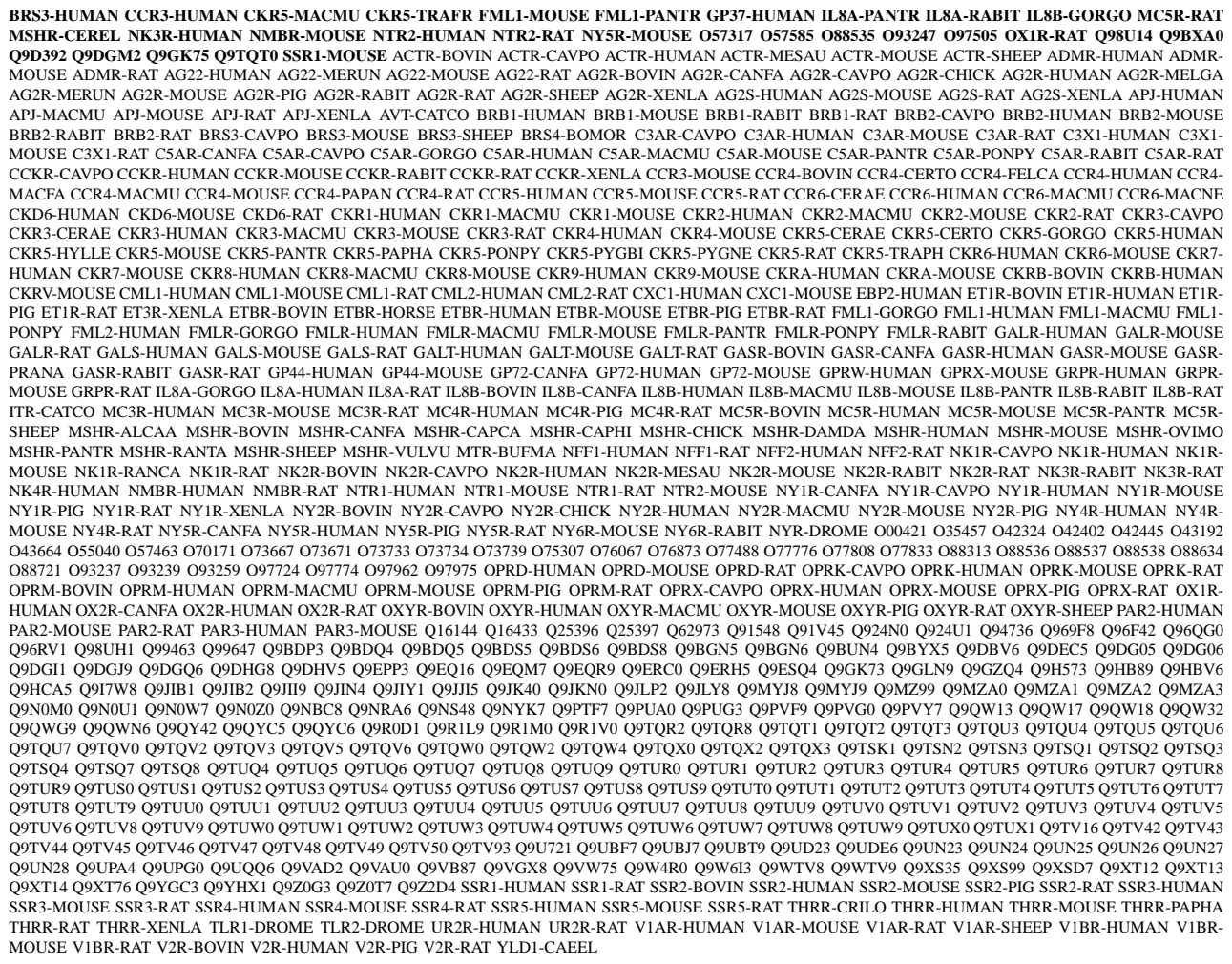 \\
\hline Hormone & 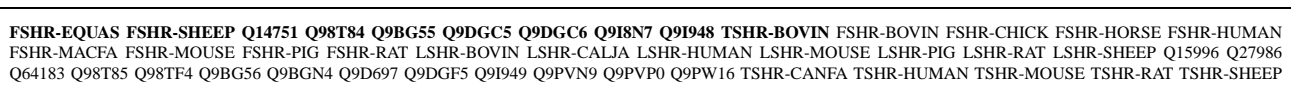 \\
\hline Rhodopsin & 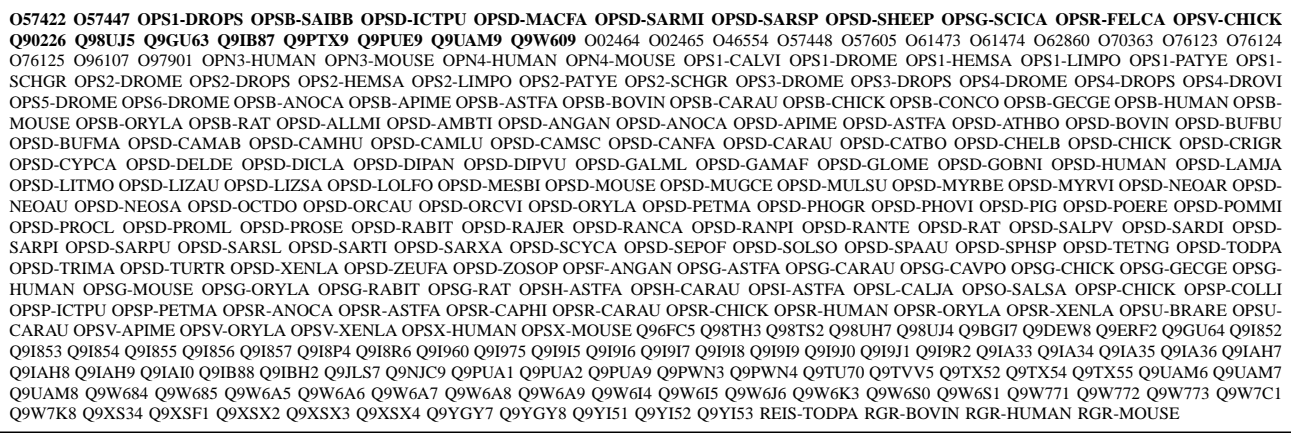 \\
\hline Olfactory & 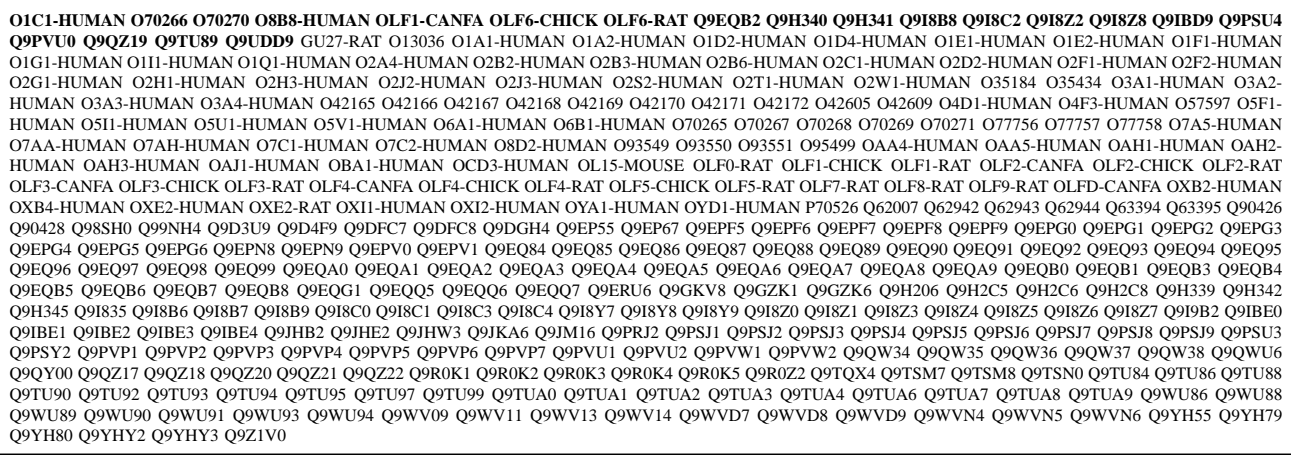 \\
\hline
\end{tabular}


TABLE 6. (Continued)

\begin{tabular}{|c|c|}
\hline Subfamily & Protein ID's \\
\hline Prostanoid & $\begin{array}{l}\text { O00326 PD2R-MOUSE PE22-MOUSE PE23-BOVIN PE23-HUMAN PE23-RABIT PF2R-MOUSE PI2R-BOVIN Q9R261 TA2R-BOVIN O00325 O15191 O35932 O46657 } \\
\text { O75228 PD2R-HUMAN PE21-HUMAN PE21-MOUSE PE21-RAT PE22-CANFA PE22-HUMAN PE22-RAT PE23-MOUSE PE23-PIG PE23-RAT PE24-HUMAN PE24-MOUSE } \\
\text { PE24-RABIT PE24-RAT PF2R-BOVIN PF2R-HUMAN PF2R-RAT PF2R-SHEEP PI2R-HUMAN PI2R-MOUSE PI2R-RAT Q9BGL8 Q9D627 Q9TU16 TA2R-CERAE TA2R- } \\
\text { HUMAN TA2R-MOUSE TA2R-RAT }\end{array}$ \\
\hline Nucleotide-like & $\begin{array}{l}\text { AA1R-BOVIN AA1R-RAT AA2A-RAT O57466 P2Y3-MELGA P2Y6-HUMAN P2YR-RAT Q99MT6 Q9ERK9 Q9H1C0 AA1R-CANFA AA1R-CAVPO AA1R-CHICK } \\
\text { AA1R-HUMAN AA1R-RABIT AA2A-CANFA AA2A-CAVPO AA2A-HUMAN AA2A-MOUSE AA2B-CHICK AA2B-HUMAN AA2B-MOUSE AA2B-RAT AA3R-CANFA } \\
\text { AA3R-HUMAN AA3R-RABIT AA3R-RAT AA3R-SHEEP GPRZ-HUMAN GPRZ-MOUSE O00398 O08766 O35811 P2UR-HUMAN P2UR-MOUSE P2UR-RAT P2Y3-CHICK } \\
\text { P2Y4-HUMAN P2Y5-CHICK P2Y5-HUMAN P2Y6-RAT P2Y8-XENLA P2Y9-HUMAN P2YR-BOVIN P2YR-CHICK P2YR-HUMAN P2YR-MELGA P2YR-MOUSE Q9BXA5 } \\
\text { Q9BXC1 Q9BYU4 Q9CPZ4 Q9DE05 Q9JJS7 Q9N1U0 Q9PU18 Q9R202 Q9W6C4 }\end{array}$ \\
\hline
\end{tabular}

\section{ACKNOWLEDGMENTS}

The authors wish to thank the anonymous referees for the valuable comments that have improved the quality and the presentation of this work.

\section{REFERENCES}

Almeida, J.S., and Vinga, S. 2002. Universal sequence map (USM) of arbitrary discrete sequences. BMC Bioinformatics 3(6).

Altshul, S.F., Gish, W., Miller, W., Myers, E.W., and Lipman, D.J. 1990. Basic local alignment tool. J. Mol. Biol. 215, 403-410.

Bailey, T.L., and Elkan, C. 1994. Fitting a mixture model by expectation maximization to discover motifs in biopolymers. 2nd Int. Conf. on Intelligent Systems for Molecular Biology, 28-36.

Bailey, T.L., and Gribskov, M. 1998. Combining evidence using p-values: Application to sequence homology searches. Bioinformatics 14, 48-54.

Bishop, C.M. 1995. Neural Networks for Pattern Recognition, Oxford University Press, New York.

Brāzma, A., Jonasses, I., Eidhammer, I., and Gilbert, D. 1998. Approaches to the automatic discovery of patterns in biosequences. J. Comp. Biol. 5(2), 277-303.

Dayhoff, M.O., Schwartz, R.M., and Orcutt, B.C. 1978. Atlas of Protein Sequence and Structure, Vol. 5, Natl. Biomed. Res. Found., Washington, DC.

Dempster, A.P., Laird, N.M., and Rubin, D.B. 1977. Maximum likelihood from incomplete data via the EM algorithm. J. Roy. Statist. Soc. B 39, 1-38.

Durbin, R., Eddy, S., Krough, A., and Mitchison, G. 1998. Biological Sequence Analysis: Probabilistic Models of Protein and Nucleic Acid, Cambridge University Press, New York.

Foresse, F.D., and Hagan, M.T. 1997. Gauss-Newton approximation to Bayesian regularization. Proc. 1997 Int. Joint Conf. on Neural Networks, 1930-1935.

Henikoff, S.S., and Henikoff, J.G. 1994. Protein family classification based on searching a database of blocks. Genomics 19, 97-107.

Hertz, G.Z., and Stormo, G.D. 1999. Identifying DNA and protein patterns with statistically significant alignments of multiple sequences. Bioinformatics 15(7/8), 563-577.

Hofmann, K., Bucher, P., Falquet, L., and Bairoch, A. 1999. The PROSITE database, its status in 1999. Nucl. Acids Res. 27, 215-219.

Horn, F., Weare, J., Beukers, M.W., Hörsch, S., Bairoch, A., Chen, W., Edvardsen, O., Campagne, F., and Vriend, G. 1998. GPCRDB: An information system for G protein-coupled receptors. Nucl. Acids Res. 21(1), 227-281.

Hughey, R., and Krogh, A. 1996. Hidden Markov models for sequence analysis: Extension and analysis of the basic method. CABIOS 12(2), 95-107.

Jaakkola, T., Diekhans, M., and Haussler, D. 2000. A discriminative framework for detecting remote protein homologies. J. Comp. Biol. 7(1-2), 95-114.

Karchin, R., Karplus, K., and Haussler D. 2002. Classifying G-protein coupled receptors with support vector mahcines. Bioinformatics 18(1), 147-159.

Karplus, K., Barrett, C., and Hughey, R. 1998. Hidden Markov models for detecting remote protein homologies. Bioinformatics 14(10), 846-856.

Lawrence, C.E., Altschul, S.F., Boguski, M.S., Liu, J.S., Neuwland, A.F., and Wootton, J.C. 1993. Detecting subtle sequence signals: A Gibbs sampling strategy for multiple alignment. Science 226, 208-214.

Logan, B., Moreno, P., Suzek, B., Weng, Z., and Kasif, S. 2001. A study of remote homology detection. Technical report CRL 2001/05, Cambridge Research Laboratory. 
Ma, Q., and Wang, J.T.L. 2000. Application of Bayesian neural networks to protein sequence classification. ACM SIGKDD Int. Conf. on Knowledge Discovery and Data Mining, 305-309.

MacKay, D.J.C. 1992. Bayesian interpolation. Neural Comp. 4, 415-447.

Rigoutsos, I., Floratos, A., Parida, L., Gao, Y., and Platt, D. 2000. The emergency of pattern discovery techniques in computational biology. Metabolic Eng. 2, 159-177.

Vapnik, V.N. 1979. Estimation of Dependencies Based on Empirical Data, Nauka, Birmingham, AL.

Wang, J.T.L., Ma, Q., Shasha, D., and Wu, C.H. 2001. New techniques for extracting features from protein sequences. IBM: Systems Journal 40(2), 426-441.

Wu, C.H., Zhap, S., Chen, H.L., Lo, C.J., and McLarty, J. 1996. Motif identification neural design for rapid and sensitive protein family search. CABIOS 12(2), 109-118.

Address correspondence to:

Konstantinos Blekas

Department of Computer Science and Biomedical Research Institute-FORTH

University of Ioannina

GR-45110 Ioannina, Greece

E-mail: kblekas@cs.uoi.gr

\begin{abstract}
Author
Right running head okay as shown (short title)?
\end{abstract}

Pub/Author

Appendix tables (Tables 4-6) were able to be picked up from electronic file for manipulation. So, these tables are supplied as type rather than art. Okay? 